\title{
Interactive Visual Simulation of Dynamic Ink Diffusion Effects
}

\author{
Shibiao Xu*,1, Xing Mei ${ }^{\dagger, 1}$, Weiming Dong ${ }^{\ddagger, 1}$, Zhiyi Zhang ${ }^{\S, 2}$, and Xiaopeng Zhang ${ }^{\llbracket, 1}$ \\ ${ }^{1}$ NLPR-LIAMA-Digital Content Technology Research Center, Institute of Automation, CAS, Beijing, China \\ ${ }^{2}$ Science Art Center, Institute of Automation, CAS, Beijing, China
}
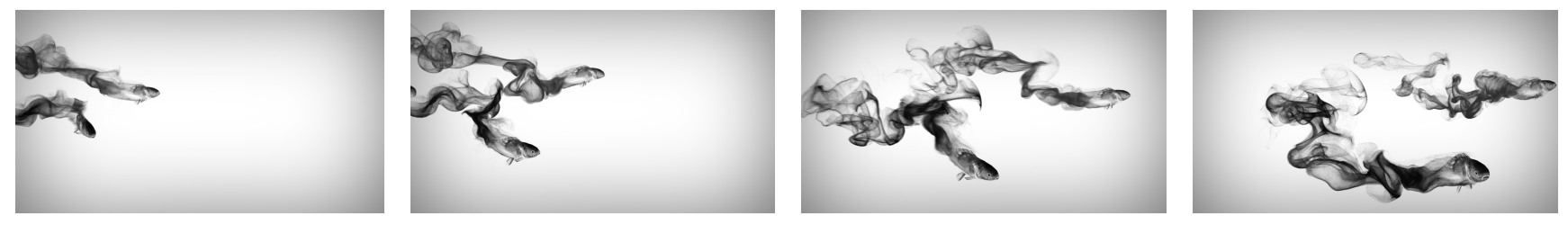

Figure 1: An example of ink-made fish animation created with our method. Two fish are swimming in water freely, and the fishtails swing in high speed. Ink flows emit from the fishtails at the same time, then the attractive dynamic ink diffusion effects can be observed.

\section{Abstract}

This paper presents an effective method that simulates the ink diffusion process with visual plausible effects and real-time performance. Our algorithm updates the dynamic ink volume with a hybrid grid-particle representation: the fluid velocity field is calculated with a low-resolution grid structure, while the highly detailed ink effects are controlled and visualized with the particles. We propose an improved ink rendering method with particle sprites and motion blur techniques. The simulation and the rendering processes are efficiently implemented on graphics hardware for interactive frame rates. Compared to traditional simulation methods that treat water and ink as two mixable fluids, our method is simple but effective: it captures various ink effects such as pinned boundary [Chu and Tai 2005] and filament pattern [Shiny et al. 2010] with real-time performance; it allows easy interaction with the artists; it includes basic solid-fluid interaction. We believe that our method is attractive for industrial animation and art design.

CR Categories: I.3.7 [Computer Graphics]: Three-Dimensional Graphics and Realism-Animation;

Keywords: Ink Diffusion, Industrial Animation, Interaction, Motion Blur

\footnotetext{
*e-mail: shbxu@nlpria.ac.cn

†e-mail:xmei@nlpr.ia.ac.cn

†e-mail:wmlake@gmail.com

$\S$ e-mail:zhiyi.zhang@ia.ac.cn

ףe-mail:xiaopeng.zhang@ia.ac.cn
}

\section{Introduction}

Ink is unique among all kinds of pigments; different concentrations of ink exhibit different intensity levels [Kunii et al. 2001]. Ink in its solid form is a mixture of soot and glue, and it is grinded together with water [Swider et al. 2003]. The most attractive visual characteristic of ink is its dynamic diffusion effects when ink drops into the water, the ink drops spread out and mix together with the water, and the mix of ink and water molecules makes the fluid look delicate and beautiful. This phenomenon is of great interest for art productions. In this paper, we aim at generating visual plausible simulation of dynamic ink diffusion with high performance, an example of applying these special effects in computer animation is shown in Figure 1.

When ink drops spread in water, realistic integration occurs not only with the fluid surface but also with the internal motion of fluid itself It is costly or sometimes impossible to capture these special flow effects from real scenes in various conditions. Therefore, synthetic method such as fluid simulation [Bridson et al. 2006] has been extensively used in computer animation industry.

Current fluid simulations fall into two major categories: the Euler method and the Lagrange method. The Euler method [Selle et al. 2005] [Enright et al. 2002] [Nguyen et al. 2002] focuses on fixed point in space grid and see how measurements of fluid quantities. Instead of fixed points, the Lagrange method [Müller et al. 2003] [Becker and Teschner 2007] [Clavet et al. 2005] treats the fluid as a particle system. Despite both the methods are useful, some major drawbacks remain. A three dimensional computational grid in Euler approach requires a lot of memory, and it will bring excessive numerical diffusion, so it is difficult to simulate high detail and large scale phenomena. Particles method such as SPH [Goswami et al. 2010] [Lenaerts and Dutré 2008] can avoid the memory requirements of a three dimensional grid, but it exhibits other difficulties such as the cost of finding the nearest neighbors and tracing the interface between different fluids.

Since grids and particles have complementary strengths and weaknesses, during the last years, many hybrid methods [Kim et al. 2006] [Zhu and Bridson 2005] have been proposed to simulate fluid with high quality and a lot of details. Due to the big advancement of fluid simulation, mixable fluid phenomena become a popular topic, and multiple fluid simulations prefer to take ink diffusing in water for example. However, these methods mainly focus on the interface of different fluids and simulate the multiple fluids in common, they do not consider the attractive effects observed when ink diffuse in 
water, so they cannot simulate small internal movements and smallscale features such as filament pattern [Shiny et al. 2010]. Furthermore, they are quite realistic by using many complex physical models and additional assumptions, which bring significant computation cost. Therefore, they are not suitable for computer animation which requires visual plausibility and low simulation cost. On the other hand, when it comes to simulate small-scale features of ink, every tiny movement will be put under heavy scrutiny, so it is beneficial that the animation can be efficiently simulated, edited and even stored by artist at interactive speeds. At present, there are many 3D computer graphics softwares such as SideFX Houdini and Autodesk Maya used to create computer animation, however, most of them are limited, especially in the simulation of fluid phenomena. The simulation time consumed is too long and it is difficult to simulate the special effects of ink diffusion freely. Figure 2 is an example of ink diffusion in water using Maya fluids, artist can only get it by adjusting the opacity property of fluids repeatly and increasing key frames in Maya, so it is impossible to get more internal detail of ink flow, what is more, in this traditional method, the emitters must be static and the ink flow cannot interact with other objects, otherwise the results will be artificial, so we cannot apply this method in computer animation.

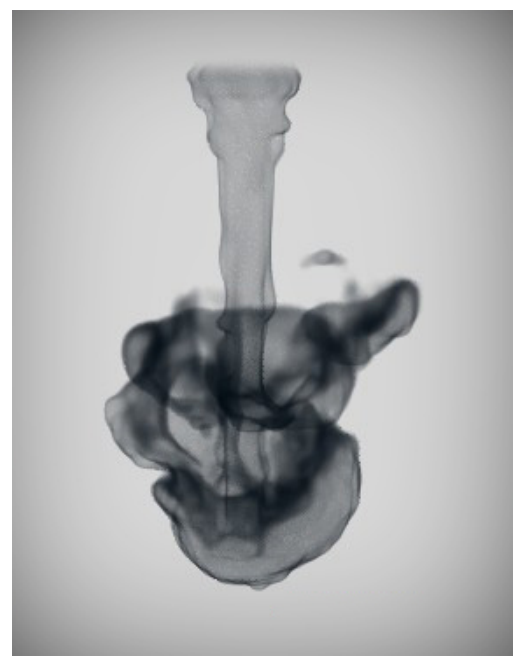

Figure 2: An example of ink diffusion in water using Maya fluids. The internal opacity of ink flow is lower than the external value.

Inspired by the existing fluid simulations, we simulate dynamic ink diffusion with a hybrid grid-particle representation. In consideration of the components of real liquid ink, we use particles to represent the ink particles, and make grid fluid as water to dissolve ink particles. In fact, the water based on grid and ink particles are emitted from the ink-made virtual objects simultaneously, our simulation only visualize the highly detailed ink effects with particles by assuming the grid water is completely transparent, but the region of grid water is real to control the advection and boundary of particles. So all the spatial interactions including coupling, incompressibility, and pressure are calculated with a low-resolution grid structure, ink particles only handle advection depending on the water's velocity field. The rendering of ink particles is crucial, because every point is rendered; it is likely to get a grainy look if there are no enough particles. Solving this problem requires a large number of particles. However, more ink particles will bring more computational time. To keep the detail plausible and smooth in real-time, we propose an improved ink rendering method with sprites rendering and motion blur technique.

The simulation is implemented on graphics hardware with real-time performance. Compare to traditional simulation methods that bring complex physical models and additional assumptions, our method is simple but effective. In our simulation, the internal detail of liquid ink is equally as important as the surface, therefore, our method can capture various ink effects such as pinned boundary and filament pattern; it allows easy interaction with the artist, thus the ink animation can be efficiently simulated, edited and even stored by artist at interactive speeds; because our method satisfies no-slip boundary conditions, which allow the fluid to slip past tangentially but not to flow through the solid, so it can handle the coupling between ink and dynamic solid in high speed, and the velocity of ink particles will change over time due to the dynamic solid. We believe that our method is attractive and plausible enough for computer animation industries.

\section{Related Work}

We briefly review relevant papers, primarily in fluid simulation, that inspire us to simulate dynamic ink diffusion.

Ink simulation: several authors have worked on ink effects, but most focused on the ink dispersion in paper. Guo and Kunii [Guo and Kunii 1991] proposed the first model specifically for ink dispersion in paper using particles of different sizes. There also have many other methods to describe the dispersion phenomenon including partial differential equations (PDE) method [Kunii et al. 2001] and cellular automation [Zhang et al. 1999] method. However, these methods are only suitable for situations where ink get mixed to form blurry images without the interesting flow patterns observed in reality. Until 2005, Nelson [Chu and Tai 2005] presented a physically-based method for simulating ink dispersion in absorbent paper, which can achieve complex and realistic ink effects. They implemented a digital paint system which can achieve various effects of ink dispersion in paper in real time.

Fluid simulation: physically-based fluid simulation is often modeled using the incompressible Navier-Stokes equations. In 1996, Foster and Metaxas [Foster and Metaxas 1996], for the first time, introduced a fully three-dimensional Navier-Stokes solver for fluid simulation in computer graphics, their system works nicely, but the explicit time stepping scheme leads to instability. In 1999, Jos Stam [Stam 1999] moved the approach of Foster and Metaxas forward tremendously by presenting a semi-Lagrangian method for solving the Navier-Stokes equations, which is unconditionally stable for any time step. Due to its stability for large time steps and the linear interpolation in first-order, semi-lagrangian velocity advection comes with built-in dissipation, which may remove small scale and even large scale motions. In 2001, vorticity confinemen$\mathrm{t}$ [Fedkiw et al. 2001] was added to generate a small scale fluid rolling motion. Back and Forth Error compensation and Correction (BFECC) [Kim et al. 2007] can also be applied to reduce dissipation and diffusion encountered in a variety of advection steps, such as velocity and density. What is more, there are many high order methods for improving the accuracy in the advections, however, the BFECC can be implemented more easily and exhibits second-order accuracy in both space and time. From then on, a wide variety of methods including Euler methods [Selle et al. 2005] [Enright et al. 2002] [Nguyen et al. 2002] and Lagrangian methods [Müller et al. 2003] [Becker and Teschner 2007] [Clavet et al. 2005] have been developed. Compare to Lagrangian method, Euler method is easier to analytically work with spatial derivatives like the pressure gradient and viscosity term, but it has difficulties in the advection term with large time steps. Therefore, the Particle-in-cell (PIC) [Harlow 1963] method and the Fluid-Implicit-Particle (FLIP) [BRACKBILL and RUPPEL 1986] method are proposed to handle advection with particles instead of grid. The major problem with PIC and FLIP is the excessive numerical diffusion in the fluid due to aver- 
age the fluid variables repeatedly. In 2005, Zhu and Bridson [Zhu and Bridson 2005] developed a fluid solver that combines the PIC method and the FLIP method to generate sands animation efficiently, in which only particles represent the fluid and grid is auxiliary for calculations. Giving some necessary important improvements, we also combine the approaches of grids and particles to achieve plausible ink simulation.

Due to the big advancement of fluid simulation technology, the interactions of multiple fluids have been a popular topic in graphics to simulate. Zhu [Zhu et al. 2006] [Zhu et al. 2007] simulates a pair of miscible fluids by using a Lattice Boltzmann Method (LBM), however, when the Reynolds number is too high, LBM is unstable and cannot satisfy the divergence-free condition of fluids. In addition, LBM-based approaches cannot be easily integrated into the conventional fluid simulation pipeline. We follow the semiLagrangian method of Stam [Stam 1999] which is the standard operator-splitting method for solving the Navier-Stokes equations to get divergence-free of fluid, together with vorticity confinement [Fedkiw et al. 2001] and the BFECC method [Kim et al. 2007] to mitigate the numerical dissipation of semi-Lagrangian method.

Nahyup and Jinho [Kang et al. 2010] present a hybrid approach for multiple fluid simulations by combining distance functions (Level set functions) and volume fractions. They also adopt the standard operator-splitting framework [Stam 1999] to solve the NavierStokes equations on grid. In order to trace the interface between multiple fluids, they have to modify the volume fractions of cells near the interface at every time step. What is more, material diffusion equation is needed for modeling diffusion phenomena based on volume fractions. Seung-Ho and Hyeong [Shiny et al. 2010] use a hybrid method of grid-based simulation and smoothed particle hydrodynamics to simulate molecular diffusion and attraction, they mainly focus on the small-scale phenomena that occur when a fluid spread out at the interface of another fluid, which cannot achieve the filaments in the fluid. In the simulations, they make two assumptions, one is that the mixing process is equivalent to a fluid passing through a porous medium with Darcy's Law, and the other is that they make the mixing surface as a fractal-like shape. In summary, both the approaches above cannot simulate small-feature such as the filaments of ink diffusion; they also need to cope with complex interface between every pair of fluids, and their methods are quite realistic and expensive by considering many additional physical equations and assumptions, which cannot be used in computer animation effectively. We propose a hybrid grid-particle representation based on the components of liquid ink, which can efficiently generate the special effects of ink diffusion in real time.

\section{Ink Diffusion Simulation}

Diffusion exists in all states of matter: solid, liquid, and gas. It is the movement of molecules from a region of high concentration to one of low concentration. Diffusion occurs rapidly enough to be observable in a reasonable period of time, however, only in liquids and gases. We describe the special effects of real ink diffusion in water, and then propose a novel hybrid grid-particle representation based on the visual characteristics of ink diffusion to simulate this phenomenon.

\subsection{Visual characteristics of Ink Diffusion}

Ink in its solid form is a mixture of soot and animal glue [Swider et al. 2003]. The solid is grinded together with water to get black liquid ink [Chu and Tai 2005]. The soot is composed of carbon particles and dissolves easily in water, and the animal glue is used to control the diffusion of ink flow. The process of diffusion is delicate and beautiful, to better appreciate this kind of diffusion,

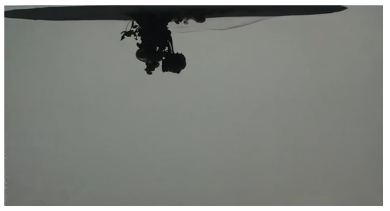

(a) Ink drops into the water.

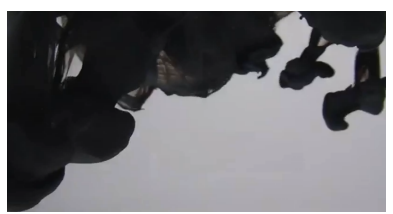

(c) Pinned boundary character.

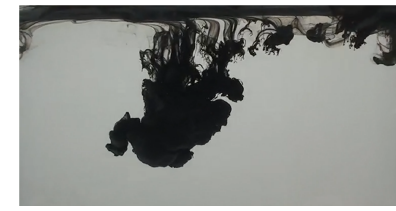

(b) Ink spreads out in the water.

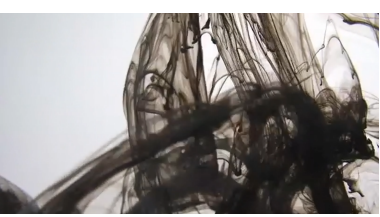

(d) Filament pattern character.
Figure 3: The process and visual effects of real ink diffusing in water.

we provide a video recorded the diffusion process; some are shown in Figure 3.

For a plausible simulation, we need to generate the essential effects of the real diffusion, which include:

Pinned boundary [Chu and Tai 2005] (Figure 3c): As ink drops into water, ink particles fast dissolves in water, due to the surface tension and water solubility of ink, the liquid ink's boundary is pinned by the water which dissolves the ink particles, and become ringlike rapidly, that means the ink particles cannot flow out the water region. This is more prominent with concentrated ink, forming boundary darkening.

Filament pattern [Shiny et al. 2010] (Figure 3d): When the ink particles diffuse in water, they spread outward in every direction to regions where they are in low concentration, the transparency will reduce over time, the texture becomes smooth and delicate as a piece of silk. Delicate ink streaks that follow the direction of the water flow will appear gradually. That is because parts of ink dissolve in water and the water molecules control the advection of the ink molecules.

\subsection{Overview of Our Method}

Construction of liquid ink model includes the components of ink and the construction of flowing mechanism in two parts. In order to meet the visual characters of ink diffusion in the simulation, one condition is that the computing method required high details cannot bring in excessive numerical dissipation; another is that the nongeometric characters of the interface between different liquid inks must be preserved naturally.

We combine Euler method and Lagrange Method to define our hybrid grid-particle representation shown in Figure 4. The representation consists of three components: a GPU-optimized Eulerian incompressible Navier-Stokes solver with vorticity confinement and BFECC, a GPU-optimized Lagrangian particles simulation, and a hardware particle sprites rendering system with motion blur. Unlike the method of Zhu and Bridson [Zhu and Bridson 2005], both particles and grid cells represent fluid in our simulation, particles are used to represent the ink molecules and corresponding grid cell is considered as water molecule. The grid water and ink particles are emitted from the same virtual objects simultaneously and then mix together. Ink particles handle advection along the water's velocity field so that they are kept in the region of grid water. In comput- 


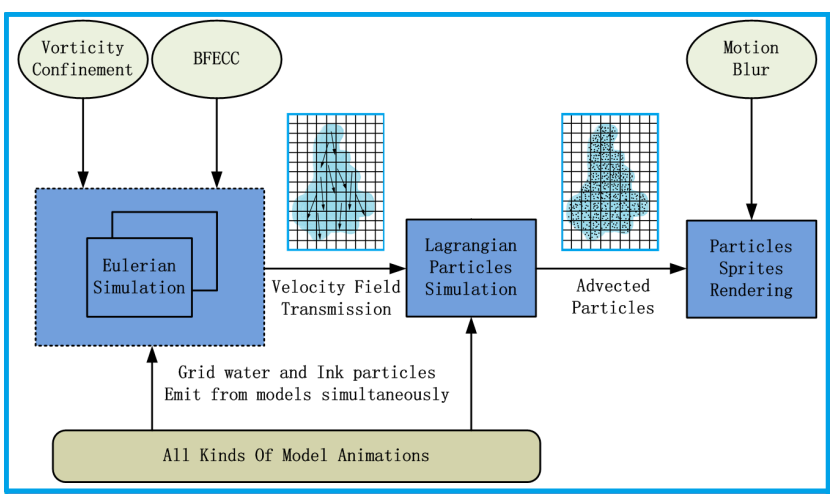

Figure 4: Overview of our method: A GPU-optimized Eulerian incompressible Navier-Stokes solver with vorticity confinement and BFECC; A GPU-optimized Lagrangian particles simulation; A hardware particle sprites rendering system with motion blur.

er animation, we only pay attention to the diffusion effect of ink particles, so we assume that the grid water is completely transparent, and it is no need to be rendered in our simulation for saving hardware resources.

\subsection{Detailed Simulation Steps}

We employ incompressible Navier-Stokes equations to simulate water on grid with vorticity confinement as follows:

$$
\begin{aligned}
& \frac{\partial \mathbf{u}}{\partial t}=-(\mathbf{u} \cdot \nabla) \mathbf{u}-\frac{1}{\rho} \nabla p+v \nabla^{2} \mathbf{u}+\mathbf{F} \\
& \nabla \cdot \mathbf{u}=0 \\
& \mathbf{f}_{v o r t}=\varepsilon(\boldsymbol{\Psi} \times \mathbf{w}) \Delta x \quad\left(\boldsymbol{\Psi}=\frac{\boldsymbol{\eta}}{|\boldsymbol{\eta}|}, \boldsymbol{\eta}=\nabla|\mathbf{w}|\right)
\end{aligned}
$$

Where $\mathbf{u}$ is the velocity of fluid, $\rho$ is the fluid density, $v$ is the kinematic viscosity, $\varepsilon$ is the strength of vorticity, $\mathbf{f}_{v o r t}$ is the vorticity confinement, $\mathbf{w}=\nabla \times \mathbf{u}$ is the divergence of the velocity, and $\mathbf{F}$ represents external forces. The numerical simulation of equation (1) and equation (2) must be stable. Thus we follow the standard operator-splitting framework of Stam [Stam 1999] to compute the velocity field on grid.

In reality, the motion of fluids typically contains all kinds of rotational flows which are vorticity. However, the excessive numerical dissipation in simulation damps out these internal details of fluids. Therefore, we apply vorticity confinement equation (3) to restore these interesting motions. Based on the classic vector calculus identity: $\nabla \cdot \nabla \times=\nabla \times \nabla \cdot$, the new velocity field with vorticity confinement is still divergence-free. What is more, we solve equation (1)-(2) using the semi-Lagrangian method with the second-order BFECC method. Because it preserves detail well, is stable for any time step size, is trivially parallelizable, and is efficient on GPU.

In our simulation we represent velocity, pressure and other quantities at cell centers, which are easy to store the variables with texture on GPU, for example, the variable for velocity is denoted $\mathbf{u}_{i, j, k}$, thus $\mathbf{u}_{i, j, k}$ is located at $(i \delta x, j \delta x, k \delta x)$, where $\delta x$ is the cell spacing along $x, y$, and $z$ axes, respectively. This is just one way to discretize the simulation domain, staggered grid method also works. In order to avoid hardware without 3D floating-point textures, we tile the $3 \mathrm{D}$ texture to a $2 \mathrm{D}$ texture. Since we want to apply the BFECC method to various advections, we use $\varphi$ to denote an advected quantity, it can be the velocity components $u, v, w$, and density $\rho$. For a given velocity field $\mathbf{u}, \varphi$ satisfies the advection equation:

$$
\varphi_{t}+\mathbf{u} \cdot \nabla \varphi=0
$$

Let $L$ be the first-order semi-Lagrangian integration steps to integrate equation (4):

$$
\varphi_{i, j, k}^{n+1}=L\left(\mathbf{u}_{i, j, k}, \varphi_{i, j, k}^{n}\right)
$$

Here, we denote $\bar{\varphi}_{i, j, k}=L\left(\mathbf{u}_{i, j, k}, \varphi_{i, j, k}^{n}\right), \mathbf{g}$ is the gravity acceleration, and $I$ is the identity matrix. Then, in each grid step time, an overview of our numerical method for solving these equations is given in Algorithm 1.

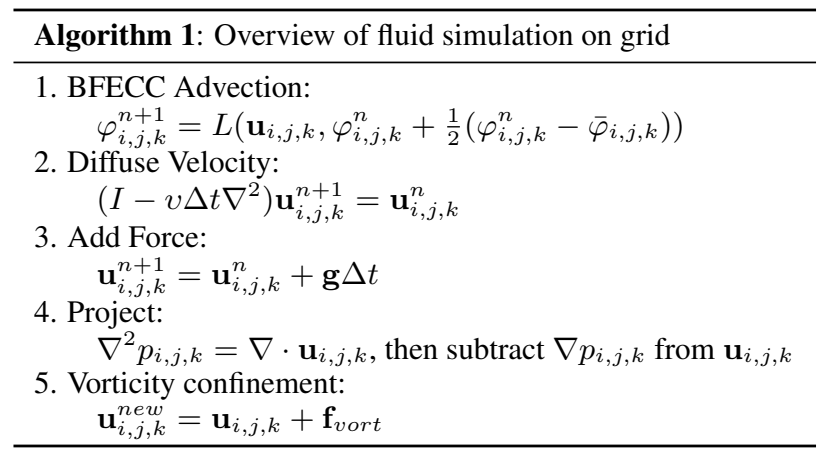

The computation of the dynamics of ink particles includes three stages: ink generation, ink movement, and ink extinction. An ink particle is generated with its initial position and velocity, and then it will mix with fluid flow. When an ink particle reaches its lifespan, we consider the ink particle to be extinct and then set its transparency to one. We employ following equations to govern the advections of ink particles according to the Lagrangian method:

$$
\begin{aligned}
& \mathbf{f}_{\text {advection }} \cdot \Delta t=\rho \cdot \mathbf{u}^{\text {new }} \\
& \mathbf{p}_{\text {particle }}^{\text {mid }}=\mathbf{p}_{\text {particle }}^{n}+\frac{1}{2} \Delta t^{\prime} \cdot \mathbf{u}_{\text {particle }}^{n} \\
& \mathbf{p}_{\text {particle }}^{n+1}=\mathbf{p}_{\text {particle }}^{\text {mid }}+\Delta t^{\prime} \cdot \mathbf{u}_{\text {particle }}^{\text {mid }} \\
& \mathbf{u}_{\text {particle }}^{n+1}=\mathbf{f}_{\text {advection }} \cdot \Delta t
\end{aligned}
$$

Where $\rho$ is the fluid density in each grid, $\mathbf{u}_{n e w}$ is the fluid velocity in each grid, $\mathbf{p}_{\text {particle }}$ is the position of every particle, $\mathbf{u}_{\text {particle }}$ is the velocity of every particle, and $\mathbf{f}_{\text {advection }}$ is the velocity field of ink particles. At each ink particle, we transfer the velocity from the corresponding grid cell that contains water molecules using equation (6). Once we have got the velocity field from the grid water, we can move the ink particles around in it. Each of particles' positions is updated using equation (7)-(8), it turns out forward Euler is sometimes adequate, but significantly better results can be obtained using a slightly more sophisticated technique such as a higher-order Runge-Kutta method, here we take second-order Runge-Kutta method as a default, it is accurate enough for our simulation. The velocity of every particle is calculated using the equation (9), which uses the grid variables to replace the velocity of particles according to the change computed on the grid. In particular, the particles every time step is not required to be same with grid every time step, so here we use $\Delta t^{\prime}$ to represent particle time step, 


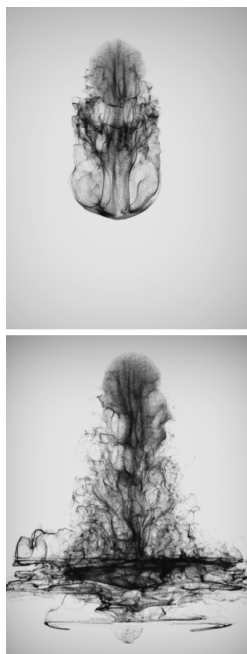

(a)

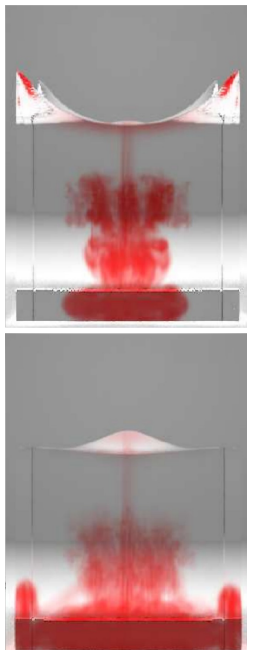

(b)

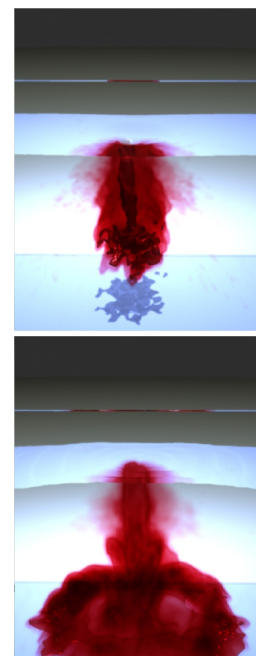

(c)
Figure 5: The comparison between our method with other methods. $a$ : Ink diffusion using our method. $b$ : Ink diffusion using the method of Nahyup. c: Ink diffusion using the method of Seung-Ho.

but it must follow the CFL condition so that a particle moves less than one grid cell in each substep.

It is crucial that the value of the velocity outside the water region must be zero, so ink particles cannot flow out the water region; this also means the region of grid water limits the simulation boundary of ink particles, which PIC and Flip cannot achieve due to the auxiliary character of grid. In respect that the velocities in adjacency grid cells are different, particles in the same grid cell tend to generate pieces of silk in the fluid flow. Especially, the internal detail effects of ink flow will still hold good when the grid is at low resolution. Figure 5A shows the process of ink diffusing in water using our method. As ink drops into the water, the surface will spread out and become more transparent at last, which make the internal fluid effects more obvious, and we can product the special effects of pinned boundary and filament pattern. Since other part of water without ink particles is negligible in computer animation, we simulate only the water flow mixed with ink particles.

We actually do an obvious comparison between our method with other methods in Figure 5. Figure 5B and Figure 5C are the simulation results using the methods of Nahyup [Kang et al. 2010] and Seung-Ho [Shiny et al. 2010] respectively. We got around 30 frames per second with five million particles under our gridparticle representation. Compare to the methods of Nahyup [Kang et al. 2010] and Seung-Ho [Shiny et al. 2010] which need at least some seconds per frame, our method is more efficient. From the effects generated point of view, we can observe that our method can achieve more special internal fluid effects of ink flow and complex fluid surface naturally. In reality, ink particles should spread out and be transparent over time, which is also required in computer animation; otherwise we cannot see the whole scene at last. Therefore, our simulation is plausible enough for art productions and it permits high-level control for artist at interactive speeds.

\section{Coupling With Solid}

In the simulation, the solid-fluid interaction must satisfy no-slip boundary conditions and pure Neumann pressure boundary conditions, allowing the fluid to slip past tangentially but not to flow through the solid. The coupling between fluid and the static container is a basic solid-fluid interaction, which assumed that the fluid domain is restricted by the container based on the simulation grid, thus the normal component of velocity and the normal pressure derivative are required to be zero at the boundaries:

$$
\begin{aligned}
& \mathbf{u} \cdot \mathbf{n}=0 \\
& \frac{\partial p}{\partial \mathbf{n}}=0
\end{aligned}
$$

However, in computer animations, the fluid domain should be unrestrained and may change over time due to the presence of dynamic obstacles in the scenes. In general, we need the normal component of the fluid velocity to match the normal component of the solid's velocity, so that the relative velocity has zero normal component:

$$
\mathbf{u} \cdot \mathbf{n}=\mathbf{u}_{\text {solid }} \cdot \mathbf{n}
$$

In practice, the velocities of dynamic obstacles are used to solve for divergence and vorticity in our simulation, we check whether the neighbor contains a solid obstacle, if it does, we replace the value stored in the fluid's velocity field with the obstacle's velocity. We apply a similar way for pressures solve using the pressure value from the center fluid cell in place of the neighbor solid cell's pressure value.

Because we cannot always solve the Navier-Stokes equations to convergence, we explicitly enforce the no-slip boundary condition immediately, following the solution of the velocity. We correct the fluid's velocity component in the direction normal to the boundary, with the corresponding component of the neighbor obstacle's velocity, so that the normal relative velocity is zero. Figure 6 shows the interaction of ink and dynamic obstacle; here we approximate the obstacle with a basic ball which can be described with an implicit equation.

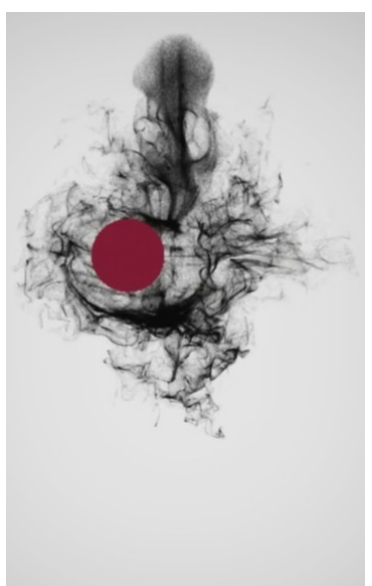

(a)

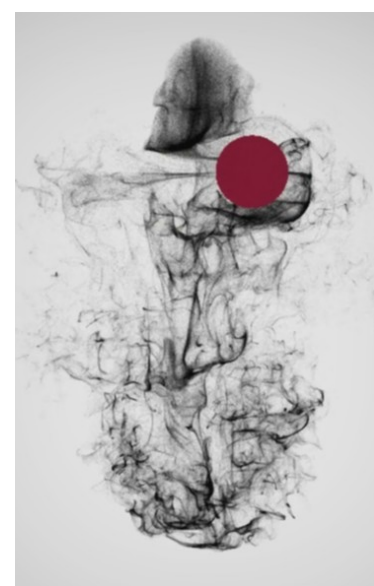

(b)
Figure 6: The interaction of ink flow and dynamic obstacle. The ink-solid interaction satisfies no-slip boundary conditions, the velocity of ink particles will change over time due to the dynamic solid. 


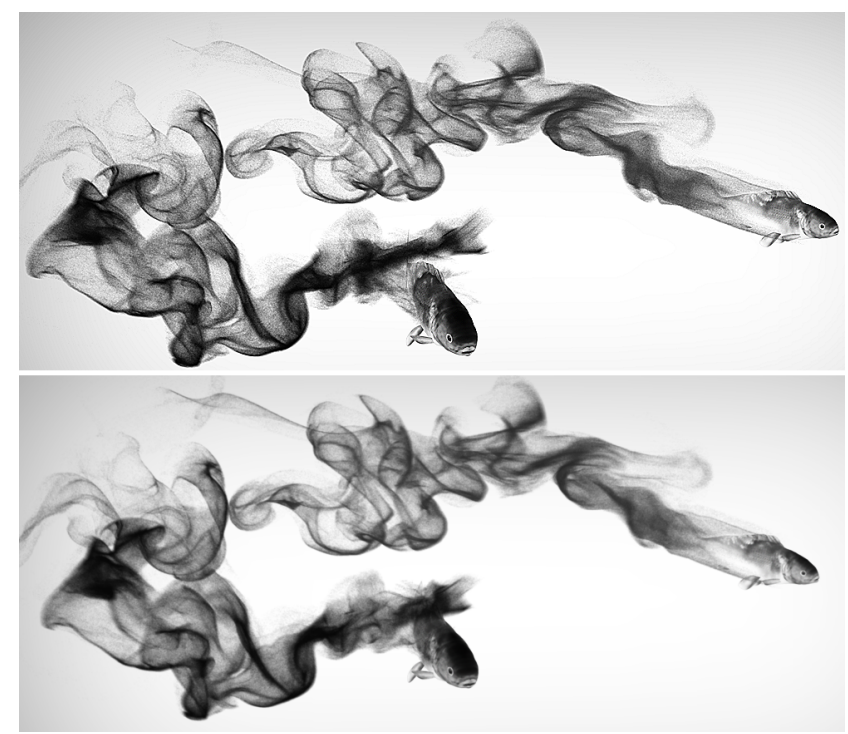

Figure 7: The effect of motion blur technique. The upper part is the simulation without motion blur. It can improve look of rapidly moving ink particles by eliminating the gaps between them as the lower part shown.

\section{Motion Blur Based Ink Rendering}

We take advantage of the hardware support, render the ink particles with texture mapping and alpha blending, and achieve more plausible results and real time as well. In our implement, each ink particle is rendered as a particle sprite which is always window-aligned. Compare to other rendering method such as volumetric fluid rendering, particles sprites rendering for particles is better choice, it can get good effects efficiently. However, there is a major problem for particles sprites rendering, it is very easy to get a grainy look which we do not want. Instead of generating more particles, we employ motion blur technique for particles rendering to smooth the final simulation and achieve better animation effects. To speed up the simulation, we save several positions along an ink particle's movement. Each ink particle has a head pointer, which is the current position, and a tail pointer, which is the previous position. In the implement, we interpolate new particles between current and previous particle position, and their colors will fade in order. Motion blur can improve look of rapidly moving ink particles by eliminating the gaps between them as Figure 7 shown. We can observe that the grainy look of animation is very obvious without motion blur as the upper part of Figure 7 shown.

\section{Implementation and Results}

We have implemented a standalone simulation system running on the GPU. We use uniform grids to discretize the simulation space, and the ink particles time step was set 1.5 times the grid water time step. The most time-consuming is the solver of grid water, therefore, we choose to implement our method on graphics hardware because it is well suited to the type of computations required by grid fluid simulation. All the simulations were performed on an AMD PC with a NVIDIA GeForce GTX 460 graphics card. Our experiments have shown the time consuming and corresponding analysis in Table 1. Firstly, the frame rates will drop rapidly when the particles number is six million, it is concerned with the performance of graphics card; secondly, less grid resolution and particle number will lead to artificial simulation results; finally, more grid resolution and particle number will need more computational resource and only get a few improvements. Therefore, we typically use five million particles and $50 \times 50 \times 50$ grid, which work best to get the special effects. Next, We will review the examples of our method one by one.

\begin{tabular}{lccc}
\hline & Four million & Five million & Six million \\
\hline Grid: $25 \times 25 \times 25$ & $45 \mathrm{fps}$ & $36 \mathrm{fps}$ & $30 \mathrm{fps}$ \\
Grid:50 $550 \times 50$ & $33 \mathrm{fps}$ & $30 \mathrm{fps}$ & $25 \mathrm{fps}$ \\
Grid: $75 \times 75 \times 75$ & $25 \mathrm{fps}$ & $21 \mathrm{fps}$ & $10 \mathrm{fps}$ \\
\hline
\end{tabular}

Table 1: Performance analysis with different grid resolution and particles number. In our simulation, we typically use five million particles and $50 \times 50 \times 50$ grid, other resolution and particles number differences can be chosen as well, but our experiments have shown that these settings work best to get the special effects.

In the first example, we simulate the process of ink diffusing in water in a containter based on the grid; we can observe that ink particles emit from a virtual ball in Figure 5A, the fluid surface will spread out and become more transparent at last, making the internal fluid effects more obvious. It is plausible enough for art productions.

In the second example, we consider the interaction between ink particles and dynamic obstacle as Figure 6 shown, the velocity of ink particles change over time due to the dynamic solid ball, and the fluid cannot flow through the solid according to no-slip boundary conditions and pure Neumann pressure boundary conditions.

In the last example, we combine particle sprites rendering and motion blur technique to improve the rendering results of ink particles. Figure 7 is a comparision about motion blur. In the upper part of Figure 7, ink particles are rendered in normal circumstances without motion blur, we can see that the grainy look of animation is very obvious. In the lower part of Figure 7, motion blur is employed, it is useful to improve look of rapidly moving ink particles by eliminating the gaps between them.

Our goal is to apply the special effects of ink diffusion in computer animation and provide the maximum degree of control and flexibility to artists; we implement our grid-particle representation within the plug-in of SideFX Houdini and Autodesk Maya. Figure 1 is an example of ink animation created with our model in Houdini. In the animation, we use six million particles to simulate the fluid. Ink particles emit from the surface of fish. Motion blur technique is used to make the fluid smooth.

\section{Conclusion}

We have developed a novel hybrid grid-particle representation to simulate smaller-scale features plausibly in real time, such as pinned boundary and filament pattern that appear when ink spreads out in water. In order to combine the attracting effects of ink diffusion with computer animation, we also introduce an extra motion blur technique to enhance the particle motion effects and make the animation plausible. Our method is powerful in ink-solid coupling. We have implemented the simulation on graphics hardware for interactive frame rates, so it allows easy interaction with the artists. We believe that our method is attractive and plausible for computer animation.

In the future, we aim to simulate more special fluid effects that can be applied in computer animation, which require simulating small amount of fluids with high quality and a lot of details. We will 
simulate the free surface effect in our ink simulation, it is useful to create more plausible effects of ink flow.

\section{Acknowledgements}

This work is supported by National Natural Science Foundation of China(No.60872120, 60902078, 61172104), Beijing Natural Science Foundation(Content-Aware Image Synthesis and Its Applications, No.4112061), French System@tic Paris-Region(CSDL Project) and ANR-NSFC(No.60911130368).

\section{References}

BeCKer, M., AND Teschner, M. 2007. Weakly compressible sph for free surface flows. In Proceedings of the 2007 ACM SIGGRAPH/Eurographics symposium on Computer animation, Eurographics Association, Aire-la-Ville, Switzerland, Switzerland, SCA '07, 209-217.

BRACKBILL, J., AND RUPPEL, H. 1986. Flip: A method for adaptively zoned, particle-in-cell calculations of fluid flows in two dimensions. Journal of Computational Physics 65, 2, 314343.

Bridson, R., FedKiw, R., AND Muller-Fischer, M. 2006. Fluid simulation: Siggraph 2006 course notes. In ACM SIGGRAPH 2006 Courses, ACM, New York, NY, USA, SIGGRAPH '06, 1-87.

ChU, N. S.-H., AND TAI, C.-L. 2005. Moxi: real-time ink dispersion in absorbent paper. ACM Trans. Graph. 24 (July), 504-511.

Clavet, S., Beaudoin, P., And Poulin, P. 2005. Particlebased viscoelastic fluid simulation. In Proceedings of the 2005 ACM SIGGRAPH/Eurographics symposium on Computer animation, ACM, New York, NY, USA, SCA '05, 219-228.

Enright, D., Marschner, S., AND Fedkiw, R. 2002. Animation and rendering of complex water surfaces. ACM Trans. Graph. 21 (July), 736-744.

FEDKIW, R., StaM, J., AND Jensen, H. W. 2001. Visual simulation of smoke. In Proceedings of the 28th annual conference on Computer graphics and interactive techniques, ACM, New York, NY, USA, SIGGRAPH '01, 15-22.

Foster, N., And Metaxas, D. 1996. Realistic animation of liquids. Graph. Models Image Process. 58 (September), 471483.

Goswami, P., Schlegel, P., Solenthaler, B., AND PajaroLA, R. 2010. Interactive sph simulation and rendering on the gpu. In Proceedings of the 2010 ACM SIGGRAPH/Eurographics Symposium on Computer Animation, Eurographics Association, Aire-la-Ville, Switzerland, Switzerland, SCA '10, 55-64.

GuO, Q., AND KunII, T. L. 1991. Modeling the diffuse paintings of sumie. In Modeling in Computer Graphics. Proceedings of the IFIP WG 5.10 Working Conference (Tokyo, apr 1991), SpringerVerlag, Tokyo · Berlin · Heidelberg, T. L. Kunii, Ed., IFIP Series on Computer Graphics, 329-338.

HARLOW, F. H. 1963. The particle-in-cell method for numerical solution of problems in fluid dynamics. In Experimental arithmetic, high-speed computations and mathematics.

Kang, N., PARK, J., Yong Noh, J., And Shin, S. Y. 2010. A hybrid approach to multiple fluid simulation using volume fractions. Computer Graphics Forum 29, 685-694.
Kim, J., Cha, D., Chang, B., Koo, B., And Ihm, I. 2006. Practical animation of turbulent splashing water. In Proceedings of the 2006 ACM SIGGRAPH/Eurographics symposium on Computer animation, Eurographics Association, Aire-la-Ville, Switzerland, Switzerland, SCA '06, 335-344.

Kim, B., LiU, Y., Llamas, I., And RossignaC, J. 2007. Advections with significantly reduced dissipation and diffusion. IEEE Transactions on Visualization and Computer Graphics 13, 135-144.

Kunit, T., Nosovskij, G., And Vecherinin, Y. 2001. Twodimensional diffusion model for diffuse ink painting. International Journal of Shape Modeling 7, 45-58.

LENAERTS, T., AND DUTRÉ, P. 2008. Unified sph model for fluidshell simulations. In ACM SIGGRAPH 2008 posters, ACM, New York, NY, USA, SIGGRAPH '08, 12:1-12:1.

Müller, M., Charypar, D., And Gross, M. 2003. Particlebased fluid simulation for interactive applications. In Proceedings of the 2003 ACM SIGGRAPH/Eurographics symposium on Computer animation, Eurographics Association, Aire-la-Ville, Switzerland, Switzerland, SCA '03, 154-159.

Nguyen, D. Q., Fedkiw, R., And Jensen, H. W. 2002. Physically based modeling and animation of fire. ACM Trans. Graph. 21 (July), 721-728.

Selle, A., Rasmussen, N., And Fedkiw, R. 2005. A vortex particle method for smoke, water and explosions. ACM Trans. Graph. 24 (July), 910-914.

Shiny, S.-H., KamZ, H. R., AND KimX, C.-H. 2010. Hybrid simulation of miscible mixing with viscous fingering. Computer Graphics Forum 29, 675-683.

Stam, J. 1999. Stable fluids. In Proceedings of the 26th annual conference on Computer graphics and interactive techniques, ACM Press/Addison-Wesley Publishing Co., New York, NY, USA, SIGGRAPH '99, 121-128.

SWider, J. R., HACKLEY, V. A., AND Winter, J. 2003. Characterization of chinese ink in size and surface. Journal of Cultural Heritage 4, 3, 175-186.

Zhang, Q., Sato, Y., Ya Takahashi, J., Muraoka, K., And CHIBA, N. 1999. Simple cellular automaton-based simulation of ink behaviour and its application to suibokuga-like $3 \mathrm{~d}$ rendering of trees. Journal of Visualization and Computer Animation 10, 27-37.

ZhU, Y., AND BRIDson, R. 2005. Animating sand as a fluid. ACM Trans. Graph. 24 (July), 965-972.

Zhu, H., LiU, X., LiU, Y., AND WU, E. 2006. Simulation of miscible binary mixtures based on lattice boltzmann method. Journal of Visualization and Computer Animation 17, 403-410.

Zhu, H., BaO, K., Wu, E., And LiU, X. 2007. Stable and efficient miscible liquid-liquid interactions. In Proceedings of the 2007 ACM symposium on Virtual reality software and technology, ACM, New York, NY, USA, VRST ’07, 55-64. 